\title{
The state of ultrasound technology in the diagnosis and treatment of liver diseases now and in the future
}

\author{
Yasuharu Imai ${ }^{1}$
}

(c) The Japan Society of Ultrasonics in Medicine 2018

\section{Introduction}

The functions of ultrasound devices are constantly advancing these days along with technological innovation. For instance, when one ultrasound manufacturer develops an ultrasound device equipped with a new technology, another manufacturer will develop a device with a more advanced version of the same technology the next year. In this article, I will present the latest major technologies being used in ultrasound for the diagnosis and treatment of liver diseases, and also discuss the outlook for the future.

\section{High-resolution B-mode imaging}

Advances in software-based beamforming technology in ultrasound devices have made it possible to form narrow and uniform ultrasound beams from shallow to deep regions. Ultrasonic probes have also been improved. Electrical potentials can now be efficiently converted even in the case of faint reflected signals by piezoelectric single crystal ultrasonic probes. The above innovative technologies now make it possible to generate high-resolution images from shallow to deep regions without lowering the frame rate (Fig. 1).

However, artifacts such as multiple reflections and side lobes cannot be completely eliminated, which sometimes makes diagnosis difficult. It is hoped that further technological innovations will reduce such artifacts in the future.

Yasuharu Imai

imaiyhosp@yahoo.co.jp

1 Department of Gastroenterological Imaging and Interventional Oncology, Juntendo University Graduate School of Medicine, Tokyo, Japan

\section{Contrast-enhanced ultrasound}

The ultrasound contrast agent most commonly used in Japan today is Sonazoid (Daiichi Sankyo, Tokyo, Japan) [1]. The principles of contrast-mode imaging remain the conventional pulse inversion, amplitude modulation, and a combination of the two; thus, there has been no major innovation. But technology that has improved the image quality of B-mode ultrasound has also improved the image quality of contrast mode. As shown in Fig. 2, it is now possible to detect uniform contrast agent signals from shallow to deep regions in the Kupffer phase. In addition, it is now becoming possible to depict more detailed changes in blood flow by reducing drops in frame rate in the vascular phase.

The Japanese population has shown an upward trend in obesity in recent years. Depiction is poor not only with B-mode but also contrast mode in highly obese cases and cases of liver cirrhosis with obesity, but it is hoped that further technological innovations will make it possible to achieve better image quality.

\section{Ultrasound elastography}

Methods currently available for measuring the elasticity of tissue using diagnostic imaging are ultrasound and magnetic resonance imaging (MRI). Ultrasound elastography is somewhat inferior in terms of objectivity, but it allows for repeated measurements in a short period of time due to its convenience. The most noteworthy ultrasound elastography technique is two-dimensional shear wave elastography [2], which employs color mapping of elasticity in real time on B-mode images (Fig. 3). Consequently, it allows for more efficient measurement as it is possible to judge whether or not elasticity is being correctly measured on real-time images. However, the process involves subjective judgment based on color mapping; thus, development of a more objective measurement method is needed. 


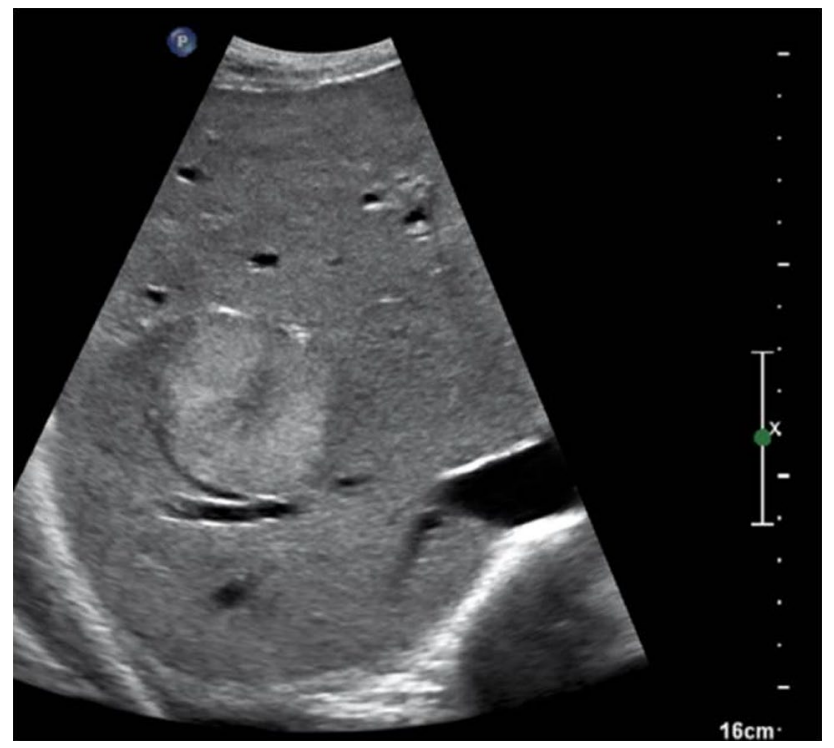

Fig. 1 High-resolution B-mode image. A high-resolution image is depicted from shallow to deep regions without lowering the frame rate

\section{Three-dimensional ultrasound}

It is now possible to acquire not only cross-sectional images but also three-dimensional volume data for ultrasound images (Fig. 4). The method for acquiring volume data has advanced from the conventional method of constructing

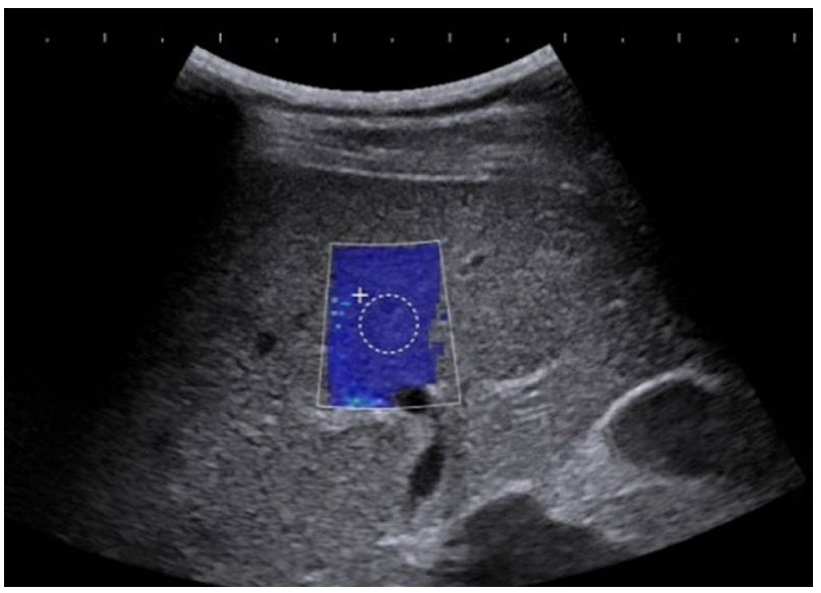

Fig. 3 Ultrasound elastography. In two-dimensional shear wave elastography, elasticity can be accurately measured at a measurable site where elasticity is color mapped in real time on the B-mode image

three-dimensional images by means of manual scan and mechanical scan to electric scanning using a two-dimensional array [3]. This is an important technology from the standpoint of improving the objectivity of examinations, but it currently lacks time resolution and its image quality is not adequate. In terms of ultrasound technology that utilizes volume data, advances in electromagnetic tracking systems, which are described below, have been remarkable.
Fig. 2 Contrast-enhanced ultrasound. A uniform contrast agent signal is detected from shallow to deep regions in the Kupffer phase

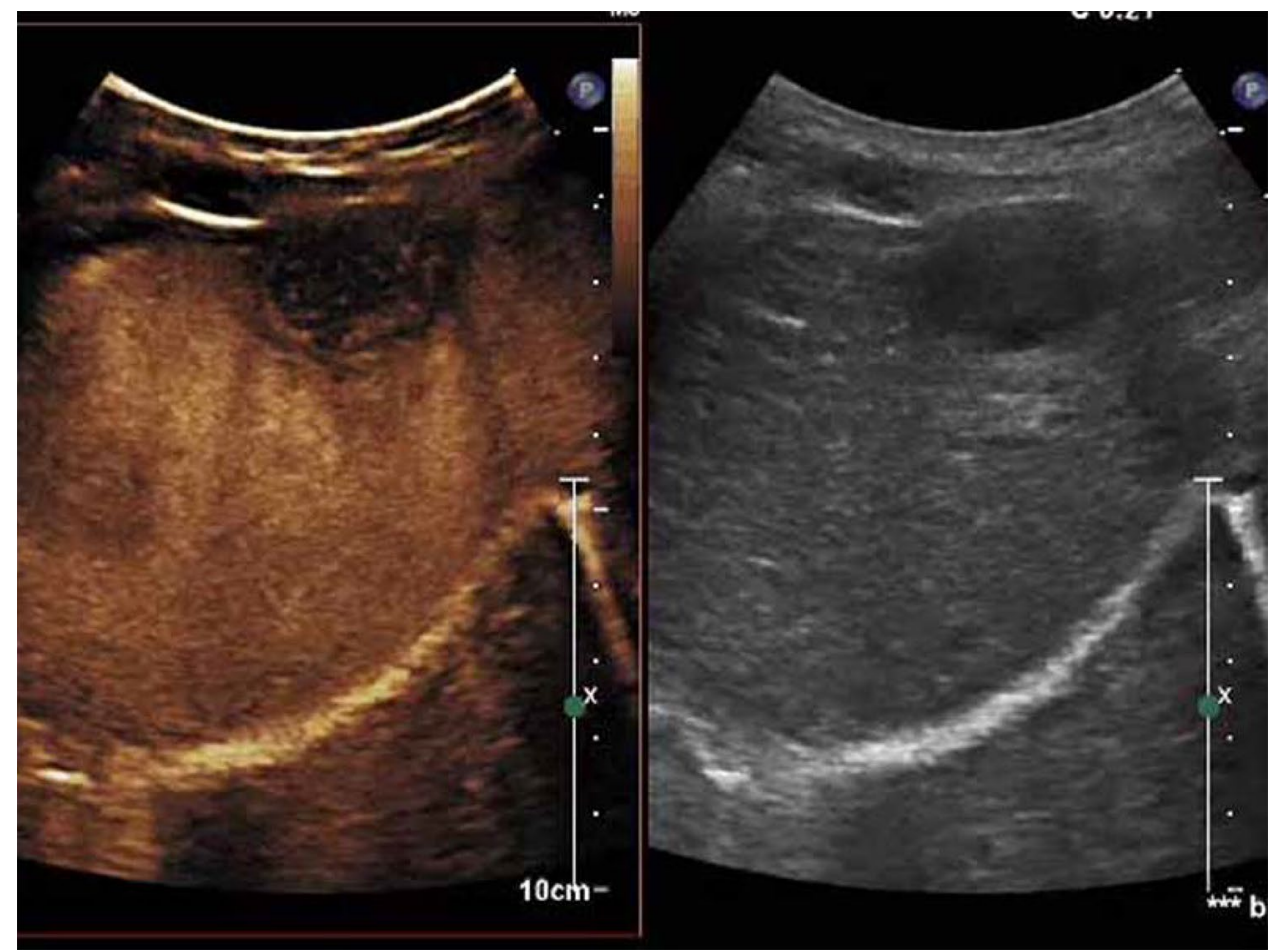


Fig. 4 Three-dimensional ultrasound. a Multi-planar reconstruction. Two sections (upper right, lower left) orthogonal to a real-time ultrasound image (upper left) are displayed at the same time. b Minimum intensity projection. Intrahepatic vessels are depicted by projecting the voxel with the lowest attenuation value on every view throughout the volume onto a 2D image
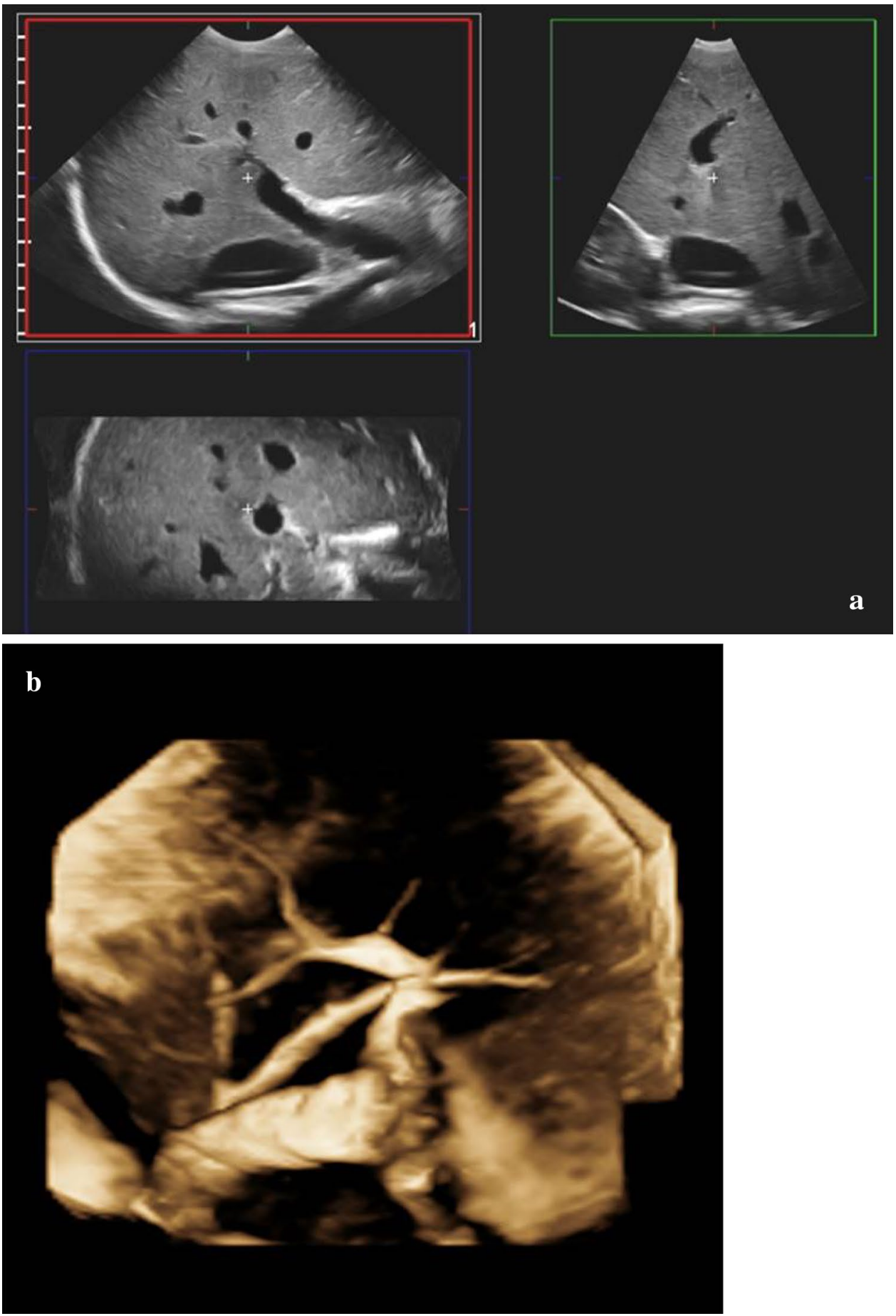

\section{Electromagnetic tracking systems}

Electromagnetic tracking systems display positional information of an ultrasonic probe or puncture needle equipped with a magnetic sensor located within a magnetic field generated by a magnetic generator in real time on an ultrasound image. Such systems are being used as fusion image ultrasound systems that display the same cross-sectional computed tomography (CT) or MRI image as the real-time ultrasound image (Fig. 5a), and as needle tracking systems that display positional information of a puncture needle in real time on an ultrasound image (Fig. 5b). Such systems are very useful for detecting liver masses depicted on CT or MRI during ultrasound examinations [4]. In local ablation therapy for liver cancer, it is also available for simulation of the puncture and monitoring of the puncture needle during the treatment, as well as assessment of the therapeutic efficacy if the conditions are good [5]. 
Fig. 5 Electromagnetic tracking system. a Fusion image ultrasound system. The upper left segment of the four-segment image displays an overlay of a real-time contrast-mode ultrasound image (upper right) and a CT multi-planar reconstruction image (lower left) which is the same cross-section as that in the real-time ultrasound image. b Needle tracking system. An ultrasound image taken during RFA performed with a magnetic sensor attached to the shaft of the RFA electrode is shown. The green dot in the center of the image displays the needle tip position of the RFA electrode. The red circle displays the expected ablated area. A hyperechoic area resulting from RFA is observed in the red circle
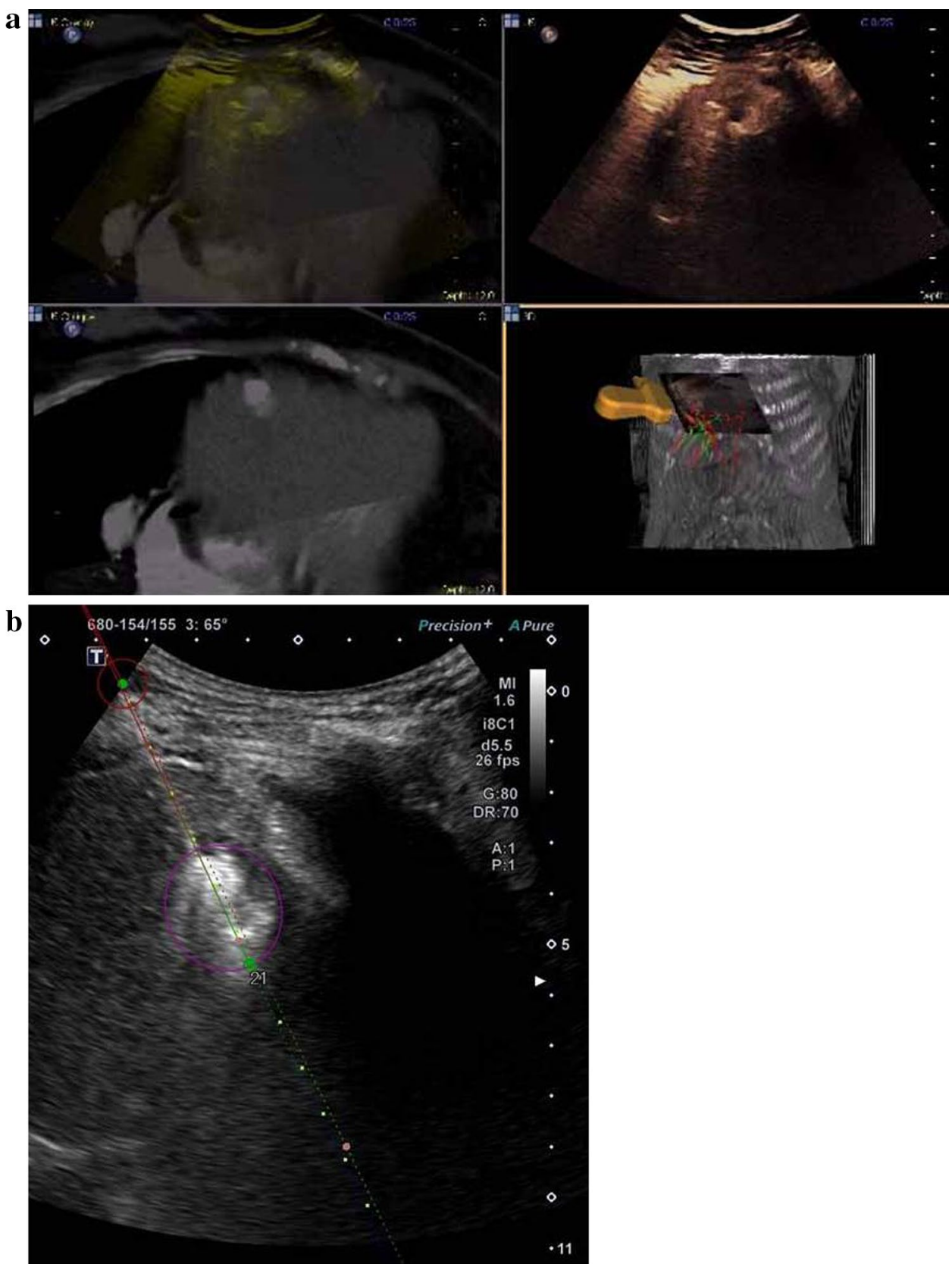

In the case of fusion image ultrasound systems, an algorithm that three-dimensionally synchronizes volume data for ultrasound images and CT/MRI images has been developed, which now allows for quite precise synchronization. However, synchronization of real-time ultrasound images and $\mathrm{CT} / \mathrm{MRI}$ reference images can be imprecise in cases where the ultrasound image quality is poor or the patient could not hold his or her breath correctly. It is hoped that further adjustments will be made to the synchronization algorithm in the future.

\section{Ultrasound-guided puncture systems}

A novel ultrasound-guided puncture system was developed in 2017 (Fig. 6a). There are both reusable and disposable ultrasound-guided puncture systems, but it is preferable that they be disposable whenever possible as they are used for invasive procedures. Free-hand puncture is the predominant method employed in the United States and Europe. The novel product allows for free-angle puncture with removal of the angle lock, in addition to five different preset puncture 

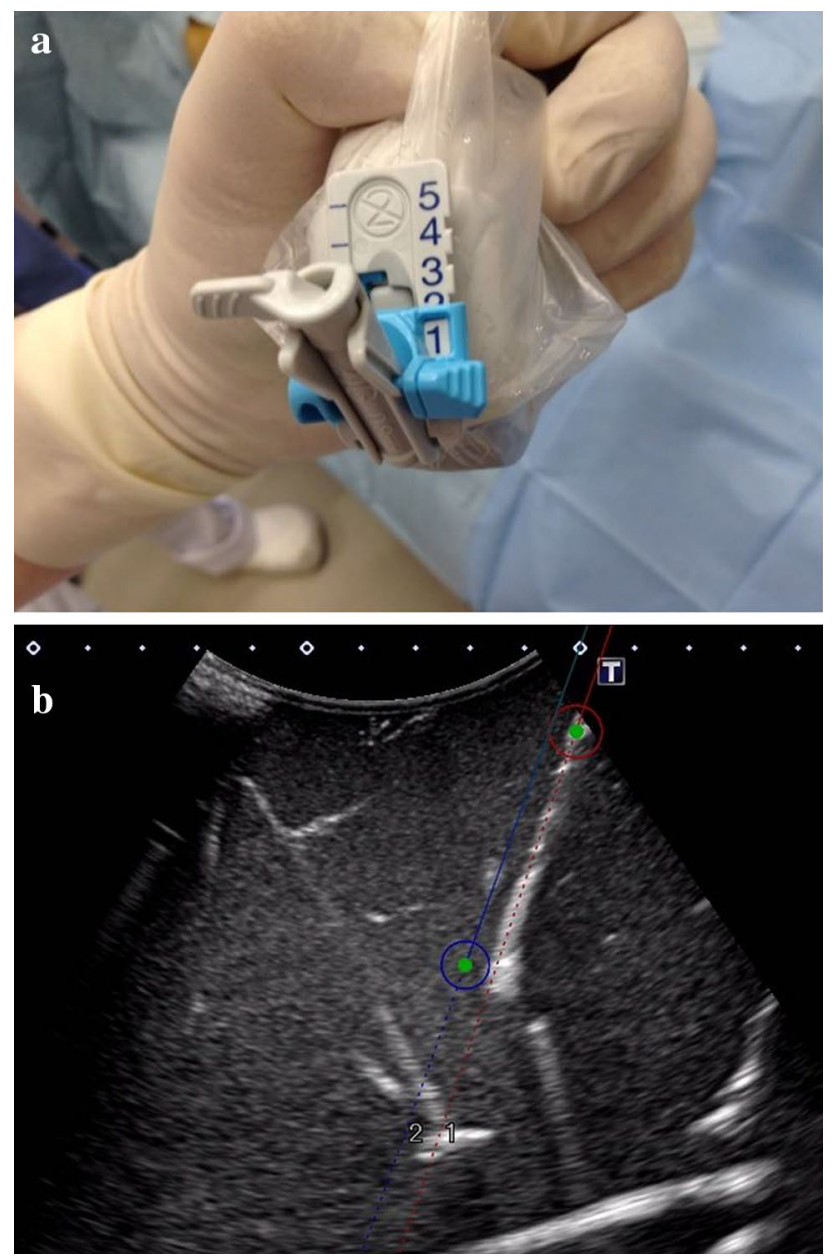

Fig. 6 Ultrasound-guided puncture system. a Novel ultrasound guidance system. A novel ultrasound guidance system has been attached to an ultrasonic probe. b Double-tracking Puncture. The RFA electrode needle (17 gauge) equipped with the no. 2 magnetic sensor is inserted along the puncture guidance line displayed by the no. 1 magnetic sensor attached to the guiding needle (14 gauge). A green dot at the center of the image displays the needle tip position of the RFA electrode

angles. Consequently, punctures can be performed at any angle without the tip of the needle deviating greatly from the ultrasound cross-sectional image. When performing freeangle puncture, a puncture guidance line is not displayed on the ultrasound image, but a puncture guidance line can be displayed during free-angle puncture by needle tracking system equipping both the puncture needle and the guiding needle with a magnetic sensor (Double-tracking Puncture) (Fig. 6b).
Despite the novel product being disposable, it has been designed to minimize the blind zone on the body surface side when inserting the needle, but it is hoped that systems that further reduce the size of the blind zone are developed.

I have given an overview of the latest major ultrasound technologies being used for the diagnosis and treatment of liver diseases, and I have also discussed the outlook for the future. I look forward to the future development of ultrasound technologies that incorporate the opinions of the people who use the technology.

\section{References}

1. Moriyasu F, Itoh K. Efficacy of perflubutane microbubbleenhanced ultrasound in the characterization and detection of focal liver lesions: phase 3 multicenter clinical trial. AJR. 2009;193:86-95.

2. Imai Y, Taira J, Okada M, et al. The close linkage between the elasticity modulus measured by real-time mapping shear wave elastography and the presence of hepatocellular carcinoma in patients with a sustained virological response to interferon for chronic hepatitis C. J Med Ultrason. 2015;42:341-7.

3. Suematsu Y, Marx GR, Triedman JK, et al. Three-dimensional echocardiography-guided atrial septectomy: an experimental study. J Thorac Cardiovasc Surg. 2004;128:53-9.

4. Kawasoe H, Eguchi Y, Mizuta T, et al. Radiofrequency ablation with the real-time virtual sonography system for treating hepatocellular carcinoma difficult to detect by ultrasonography. J Clin Biochem Nutr. 2007;40:66-72.

5. Numata K, Fukuda H, Morimoto M, et al. Use of fusion imaging combining contrast-enhanced ultrasonography with a perflubutane-based contrast agent and contrast-enhanced computed tomography for the evaluation of percutaneous radiofrequency ablation of hypervascular hepatocellular carcinoma. Eur J Radiol. 2012;81:2746-53. 